\title{
Performance Comparison of Image Retrieval Techniques using Wavelet Pyramids of Walsh, Haar and Kekre Transforms
}

\author{
Dr. H.B.Kekre ${ }^{1}$, Sudeep D. Thepade ${ }^{2}$, Akshay Maloo ${ }^{3}$ \\ ${ }^{1}$ Sr.Professor, ${ }^{2}$ Ph.D.Research Scholar and Associate Professor, ${ }^{3}$ B.Tect.(Comp) Student \\ Computer Engineering Department, SVKM's NMIMS (Deemed-to-be University) \\ Mumbai, India
}

\begin{abstract}
The paper presents performance comparison of Wavelet Pyramid based image retrieval techniques using Walsh, Haar and newly introduced Kekre wavelet transforms. Here content based image retrieval (CBIR) is done using the image feature set extracted from Wavelets applied on the image at various levels of decomposition. Here the image features are extracted by applying Wavelets on gray plane (average of red, green and blue) and color planes (red, green and blue components). The techniques Gray-Wavelets and Color-Wavelets are tested on image database having 11 categories with total 1000 images. Total 55 queries are fired on the database. The results show that precision and recall of Wavelets are better than complete transform based CBIR using Walsh and Haar transform, which proves that Wavelets give better discrimination capability in image retrieval at faster query execution speed. The Walsh and Haar Wavelets level-5 outperforms other Wavelets, because the higher level Wavelets are giving coarse color-texture features while the lower level are representing fine color-texture features which are less useful to differentiate the images in image retrieval. Color- Wavelets based CBIR have greater precision and recall than Gray-Wavelets based CBIR.
\end{abstract}

\section{Keywords}

Content Based Image Retrival (CBIR), Walsh Wavelets, Haar Wavelets, Kekre Wavelets, Texture.

\section{INTRODUCTION}

The large numbers of images has posed increasing challenges to computer systems to store, transmit and manage data effectively [3]. The storage and transmission challenge is tackled by image compression [14]. The challenge to retrieve stored images is studied in the context of image database [13], and has been attempted by many researchers worldwide from a wide range of disciplines from computer vision [15], image processing [17,18] to traditional database areas for over a decade [20,22]. Researchers are discovering that the process of locating a desired image in a large and varied collection can be a source of considerable frustration $[2,19,26]$. Problems with traditional methods of image indexing $[1-3,20]$ have led to the rise of interest in techniques for retrieving images on the basis of automaticallyderived features such as colour, texture and shape - a technology now generally referred to as Content-Based Image Retrieval (CBIR) [23,27]. "Content-based" means that the search will analyse the actual contents of the image rather than the stored metadata such as keywords, tags, descriptions associated with the image. After a decade of intensive research, CBIR technology is now beginning to move out of the laboratory and into the marketplace, in the form of commercial products like QBIC [18] and Virage [17]. However, the technology still lacks maturity, and is not yet being used on a large scale [3]. In the absence of hard evidence on the effectiveness of CBIR in practice, opinion is still sharply divided about their effectiveness in handling real-life queries in large and diverse image databases [15]. A wide range of possible applications for CBIR technology has been identified $[3,13]$. There are mainly two streams of research for image retrieval. The database community is focusing on image indexing [21] whereas image processing groups are concentrating on representing the image content in the form of some feature descriptors [16]. Most of the current image indexing practices mainly rely on color, texture or shape features. The performance of the image retrieval technique improves if these features are combined and considered together $[3,8]$. In case of color and texture features combination, the red, green and blue planes are considered separately and then some texture features are extracted from these color planes [24].

A wide variety of techniques for describing image texture have been proposed. Tuceryan and Jain [28] divided texture analysis methods into four categories: statistical, model-based, geometrical and signal processing. Here we have considered only signal processing for texture extraction and representation. If texture features are too coarse or too fine, they cannot help much in image discrimination, so the size of feature vector plays important role in performance of image retrieval [25]. In [16] novel image retrieval based on Walsh transform pyramid is proposed, which is revisited using Haar transform in [31]. The paper extends the same to newly introduced Kekre Wavelet transform and deals with performance comparison of Wavelet Pyramid based image retrieval techniques using Walsh, Haar and Kekre Wavelet transform.

Sections 2, 3 and 4 gives basics of Walsh, Haar and Kekre's Transforms respectively. The steps to generate the wavelet pyramid of transform ' $T$ ' are given in section 5. Section 6 shows the wavelet pyramid of sample image. Review of content based image retrieval is taken in section 7 and proposed image retrieval using wavelet pyramid is elaborated in section 8 . Implementation details are given in section 9. Section 10 discusses the obtained results and concluding remarks for the section 11 .

\section{WALSH TRANSFORM}

The Walsh transform matrix $[1,16,29]$ is defined as a set of $\mathrm{N}$ rows, denoted $\mathrm{Wj}$, for $\mathrm{j}=0,1, \ldots, \mathrm{N}-1$, which have the following properties:

-Wj takes on the values +1 and -1 .

$\cdot \mathrm{Wj}[0]=1$ for all $\mathrm{j}$.

-Wj $\mathrm{xWkT}=0$, for $\mathrm{j} \mathrm{k}$ and $\mathrm{Wj} \times \mathrm{WkT}=\mathrm{N}$, for $\mathrm{j}=\mathrm{k}$.

-Wj has exactly $\mathrm{j}$ zero crossings, for $\mathrm{j}=0,1, \ldots ., \mathrm{N}-1$.

-Each row $\mathrm{Wj}$ is either even or odd with respect to its midpoint. 
Walsh transform matrix is defined using a Hadamard matrix of order $\mathrm{N}$. The Walsh transform matrix row is the row of the Hadamard matrix specified by the Walsh code index, which must be an integer in the range $[0, \ldots, \mathrm{N}-1]$. For the Walsh code index equal to an integer $\mathrm{j}$, the respective Hadamard output code has exactly $\mathrm{j}$ zero crossings, for $\mathrm{j}=0,1, \ldots, \mathrm{N}-1$.

Full 2-Dimensional Walsh transform applied on an image of size $\mathrm{NxN}$ requires only additions and no multiplications. The number of additions required are $2 \mathrm{~N}^{2}(\mathrm{~N}-1)$.

\section{HAAR TRANSFORM}

This sequence was proposed in 1909 by Alfréd Haar [9]. Haar used these functions to give an example of a countable orthonormal system for the space of square-integrable functions on the real line. The study of Wavelets, and even the term "wavelet", did not come until much later [10]. The Haar wavelet is also the simplest possible wavelet. The technical disadvantage of the Haar wavelet is that it is not continuous, and therefore not differentiable. This property can, however, be an advantage for the analysis of signals with sudden transitions, such as monitoring of tool failure in machines.

The Haar wavelet's mother wavelet function $\psi(\mathrm{t})$ can be described as:

$$
\psi(t)=\left\{\begin{array}{rr}
1,0 \leq t<\frac{1}{2} \\
-1, \frac{1}{2} \leq t<1 \\
0, \text { otherwise }
\end{array}\right.
$$

and its scaling function $\varphi(t)$ can be described as:

$$
\varphi(t)=\left\{\begin{array}{l}
1,0 \leq t<1 \\
0, \text { otherwise }
\end{array}\right.
$$

\section{KEKRE TRANSFORM}

Kekre's transform $[5,19]$ matrix is the generic version of Kekre's LUV color space matrix [30]. Kekre's transform matrix can be of any size $\mathrm{NxN}$, which need not have to be in powers of 2 (as is the case with most of other transforms). All upper diagonal and diagonal values of Kekre's transform matrix are one, while the lower diagonal part except the values just below diagonal is zero. Generalized NxN Kekre's transform matrix can be given as:

$$
K_{N x N}=\left[\begin{array}{cccccc}
1 & 1 & 1 & . . & 1 & 1 \\
-N+1 & 1 & 1 & . . & 1 & 1 \\
0 & -N+2 & 1 & . . & 1 & 1 \\
\vdots & \vdots & \vdots & \vdots & \vdots & \vdots \\
0 & 0 & 0 & . . & 1 & 1 \\
0 & 0 & 0 & . . & -N+(N-1) & 1
\end{array}\right]
$$

The formula for generating the term $\mathrm{K}_{\mathrm{xy}}$ of Kekre's transform matrix is:

$$
K(x, y)=\left\{\begin{array}{cl}
1 & , x \leq y \\
-N+(x-1) & , x=y+1 \\
0 & , x>y+1
\end{array}\right.
$$

For taking Kekre's transform of an NxN image, the number of required multiplications are $(\mathrm{N}-1)$ and number of additions required are $2 \mathrm{~N}(\mathrm{~N}-1)$.

To generate NxN Kekre Wavelet transform matrix following steps could be followed:

a) Take $\mathrm{N} / 2 \mathrm{xN} / 2$ Kekre transform matrix

b) Repeat each column of Kekre transform matrix to get upper half part of Kekre Wavelet transform matrix (upper N/2 rows)

c) In lower half of Kekre Wavelet transform matrix take $-1,1,0,0 \ldots$ as the row number $(\mathrm{N} / 2+1)$.

d) Repeatedly shift rotate this row $(\mathrm{N} / 2+1)$ by two positions from left hand side to right hand side, to get the next row of Kekre Wavelet transform matrix.

\section{WAVELETS OF TRANSFORM ' $T$ '}

The procedure of generating Wavelets of transform ' $\mathrm{T}$ ' ( $\mathrm{T}$ Wavelets) [1,31] is shown in flowchart given in figure 1 and can be explained as given in following steps. Let INXN be the image with size $\mathrm{NxN}$ of which T-Wavelets are to be obtained and TNxN be the desired transform matrix (Haar, Walsh, Kekre transform) of size $\mathrm{NxN}$.

A. Apply transform of size $\mathrm{NxN}$ on the image of size $\mathrm{NxN}$ to get transformed image with approximation (tIA), horizontal (tIH), vertical (tIV) and diagonal (tID) components.

$$
\begin{gathered}
\mathrm{tI}_{\mathrm{NxN}}=[\mathrm{tIA}, \mathrm{tIH}, \mathrm{tIV}, \mathrm{tID}] \\
=\left[\mathrm{T}_{\mathrm{NxN}}\right]\left[\mathrm{I}_{\mathrm{NxN}}\right]\left[\mathrm{T}^{\prime}{ }_{\mathrm{NxN}}\right]
\end{gathered}
$$

B. Replace horizontal (tIH), vertical (tIV) and diagonal (tID) components with zero to get modified transformed image 'twI'.

$$
\text { tw } \mathrm{I}_{\mathrm{NxN}}=[\mathrm{tIA}, \text { Zero, Zero, Zero] }
$$

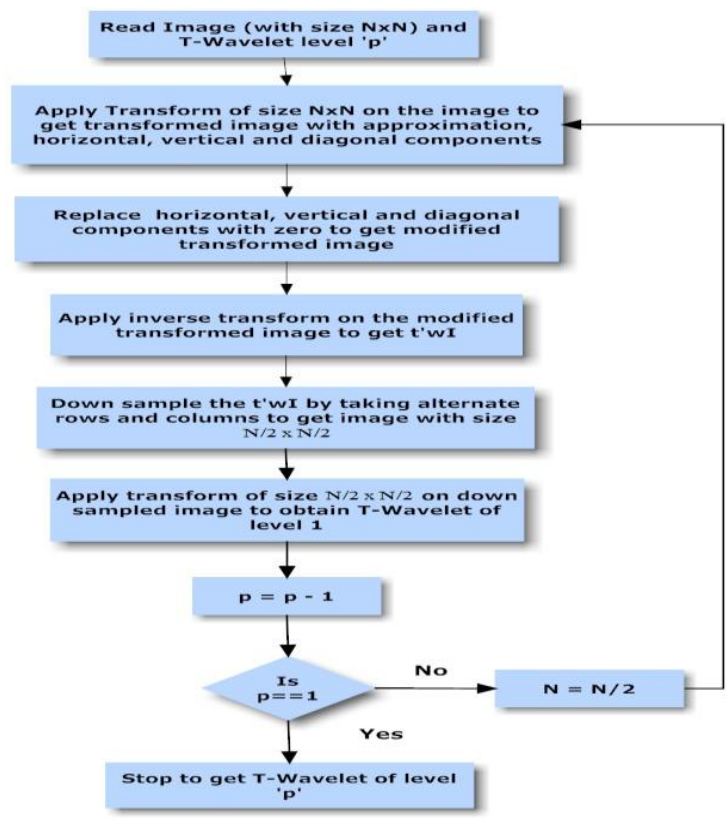

Figure1 Flowchart for generating T-Wavelets of level 'p' 
C. Apply inverse transform on the modifiedtransformed image to get t'wI.

$$
\mathrm{t}^{\prime} \mathrm{I}_{\mathrm{NxN}}=\left[\mathrm{T}^{\prime}{ }_{\mathrm{NxN}}\right]\left[\mathrm{twI}_{\mathrm{NxN}}\right]\left[\mathrm{T}_{\mathrm{NxN}}\right]
$$

D. Down-sample the result of step'c' (t'wI) by taking alternate rows and columns to get imagewith size $\mathrm{N} / 2 \mathrm{xN} / 2$.

$$
\mathrm{dtI}_{\mathrm{N} / 2 \times \mathrm{N} / 2}=\text { downsample }\left(\mathrm{t}^{\prime} \mathrm{wI}_{\mathrm{N} \times \mathrm{N}}\right)
$$

E. Apply transform of size $\mathrm{N} / 2 \mathrm{xN} / 2$ on down-sampled image ( $\mathrm{dtI}_{\mathrm{N} / 2 \times \mathrm{N} / 2}$ ) to get theT-Wavelet of level-1.

$\mathrm{T}$-Wavelet Level I =

$$
\left[\mathrm{T}_{\mathrm{N} / 2 \times \mathrm{N} / 2}\right]\left[\mathrm{dtI}_{\mathrm{N} / 2 \times \mathrm{N} / 2}\right]\left[\mathrm{T}_{\mathrm{N} / 2 \times \mathrm{N} / 2}\right]
$$

F. Repeat steps b to e 'P-1' times on the level $1 \mathrm{~T}$-Wavelet to get T-Wavelet of level 'P'.

\section{WAVELET PYRAMID OF TRANSFORM ' $T$ '}

The T-Wavelets of particular image, when considered together at different levels gives T-Wavelet Pyramid [1]. Here for the first level of T-Wavelet pyramid, transform is applied on the image to get approximation, horizontal, vertical and diagonal components. The approximation components of first level T-Wavelet is considered, transform is applied to it to get second level Haar Wavelet.

The T-Wavelet pyramid of a sample images are shown in figure 2 given below. Where the baby image is decomposed into three levels of T-Wavelet pyramid as T-Wavelet level-1, T-Wavelet level-2 and T-Wavelets level-3.

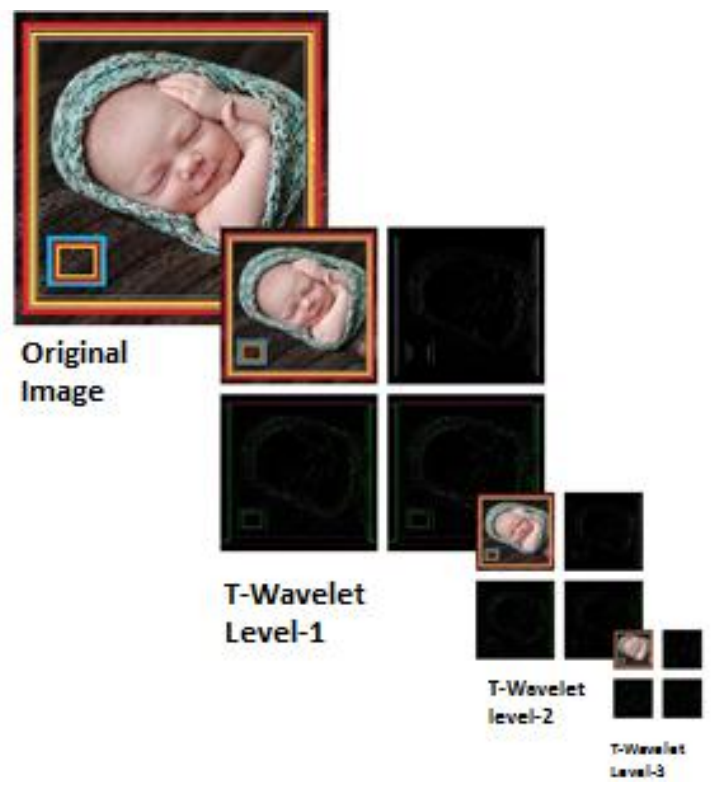

Figure 2 Different Levels of T-Wavelet Pyramid [1]

\section{CONTENT BASED IMAGE RETRIEVAL}

The earliest use of the term content-based image retrieval in the literature seems to have been by Kato et.al.[8], to describe his experiments into automatic retrieval of images from a database by color and shape feature. The typical CBIR system performs two major tasks [1],[5]. The first one is feature extraction (FE), where a set of features, called image signature or feature vector, is generated to represent the content of each image in the database. A feature vector is smaller in size than the original image, typically of the order of hundreds of elements (rather than millions). The second task of CBIR system is similarity measurement (SM) Here a distance between the query image and each image in the database using their signatures is computed so that the best matched images can be retrieved.

Most commonly Euclidian distance and correlation coefficient $[9,10]$ are used as similarity measure in CBIR. Correlation coefficient measures the cosine of the angle between two vectors and varies between 0 to 1 . When it is 1 both the vectors are aligned but their magnitude may not be same. In contrast to this Euclidian measure gives the distance between the vectors, when it is 0 not only the vectors are aligned but their magnitudes are also same. For this reason we have preferred Euclidian distance as a similarity measure.

The direct Euclidian distance between an image $\mathrm{P}$ and query image $\mathrm{Q}$ can be given as below.

$$
E D=\sqrt{\sum_{i=1}^{n}(V p i-V q i)^{2}}
$$

where, Vpi and Vqi be the feature vectors of image P and Query image $\mathrm{Q}$ respectively with size ' $\mathrm{n}$ '.

\section{IMAGE RETRIEVAL USING WAVELET PYRAMID OF TRANSFORM 'T'}

\subsection{Feature Extraction}

Here the approximate components of T-Wavelet level-1, TWavelet level-2,...., T-Wavelet level-7 are obtained for all images in the database and transforms of respective sizes are applied on them, the results are stored as feature vectors for respective image. At level-1 T-Wavelet the feature vector size is $\mathrm{N} / 2 \mathrm{xN} / 2$. At level2 T-Wavelet the feature vector size is $\mathrm{N} / 4 \mathrm{xN} / 4$ and so on. Thus the feature vectors up to level-7 T-Wavelets are extracted and feature vector database is generated.

The Gray-T-Wavelets are extracted from gray images (average of red, green and blue components is taken as gray). Then the TWavelets of Red, Green and Blue planes of images are extracted and considered as Color-T-Wavelets of respective images for various levels.

\subsection{Query Execution}

Here the feature set of T-Wavelet level-p is extracted for the query image using proposed technique of T-Wavelet generation. The obtained results are matched with T-Wavelet level-p feature vector database using Euclidian distance as similarity measure. As compared to applying complete transform on the image, the proposed method takes fewer computations to extract the feature set and gives better precision and recall values.

For image of size NxN complete Walsh needs 2N2(N-1) additions and for Walsh Wavelets of level-p the number of additions needed are $2(\mathrm{~N} / 2 \mathrm{p}) 2[(\mathrm{~N} / 2 \mathrm{p})-1]$ as the size of feature vector would be $(\mathrm{N} / 2 \mathrm{p}) \mathrm{x}(\mathrm{N} / 2 \mathrm{p})$. This gives tremendous reduction in query execution time per higher Walsh Wavelet level.

For image of size $\mathrm{NxN}$ complete Haar needs $2 \mathrm{~N} 2 \log 2(\mathrm{~N})$ additions and for Haar Wavelet of level-p the number of additions 
needed are $2(\mathrm{~N} / 2 \mathrm{p}) 2 \log (\mathrm{N} / 2 \mathrm{p})$ as the size of feature vector would be $(\mathrm{N} / 2 \mathrm{p}) \mathrm{x}(\mathrm{N} / 2 \mathrm{p})$. This gives tremendous reduction in query execution time per higher Haar Wavelet level.

For image of size $\mathrm{NxN}$ complete Kekre needs $(\mathrm{N} 2+3 \mathrm{~N}-4)$ additions with (N-4) multiplications. So by considering 1 multiplication equal to 8 additions, total number of additions needed are $(\mathrm{N} 2+11 \mathrm{~N}-36)$ and Kekre Wavelet of level-p the number of additions needed are [(N/2p) $2+11(\mathrm{~N} / 2 \mathrm{p})-36)]$. As the size of feature vector would be $\mathrm{N} / 2 \mathrm{pxN} / 2 \mathrm{p}$, this gives tremendous reduction in query execution time per higher Kekre Wavelet level.

Table 1 Complexity Analysis of generating Wavelet Pyramid

\begin{tabular}{|c|c|c|c|}
\hline \multicolumn{4}{|c|}{ Number of additions needed for NxN Image } \\
\hline & $\begin{array}{l}\text { Haar } \\
\text { Wavelet } \\
\text { Transform }\end{array}$ & $\begin{array}{l}\text { Walsh } \\
\text { Wavelet } \\
\text { Transform }\end{array}$ & $\begin{array}{l}\text { Kekre } \\
\text { Wavelet } \\
\text { Transform }\end{array}$ \\
\hline $\begin{array}{l}\text { Complete } \\
\text { Transform } \\
\text { of Image }\end{array}$ & $2 N^{2} \log _{2}(N)$ & $2 N^{2}(N-1)$ & $\left(N^{2}+11 N-36\right)$ \\
\hline $\begin{array}{l}\text { Obtaining } \\
\text { Wavelet } \\
\text { of Level-p }\end{array}$ & $\begin{array}{c}2\left(\mathrm{~N} / 2^{\mathrm{p}}\right)^{2} \\
\log \left(\mathrm{N} / 2^{\mathrm{p}}\right)\end{array}$ & $\begin{array}{c}2\left(\mathrm{~N} / 2^{\mathrm{p}}\right)^{2} \\
{\left[\left(\mathrm{~N} / 2^{\mathrm{p}}\right)-1\right]}\end{array}$ & $\begin{array}{c}{\left[\left(\mathrm{N} / 2^{\mathrm{p}}\right)^{2}+\right.} \\
\left.\left.11\left(\mathrm{~N} / 2^{\mathrm{p}}\right)-36\right)\right]\end{array}$ \\
\hline \multicolumn{4}{|c|}{ Number of additions needed for 256x256 Image } \\
\hline $\begin{array}{l}\text { Complete } \\
\text { Transform }\end{array}$ & $10,48,576$ & $3,34,23,360$ & 68,316 \\
\hline $\begin{array}{l}\text { Wavelet } \\
\text { Level-1 }\end{array}$ & $2,29,376$ & $41,61,536$ & 17,756 \\
\hline $\begin{array}{l}\text { Wavelet } \\
\text { Level-2 }\end{array}$ & 49,152 & $5,16,096$ & 4,764 \\
\hline $\begin{array}{l}\text { Wavelet } \\
\text { Level-3 }\end{array}$ & 10,240 & 63,488 & 1,340 \\
\hline $\begin{array}{l}\text { Wavelet } \\
\text { Level-4 }\end{array}$ & 2,048 & 7,680 & 396 \\
\hline $\begin{array}{l}\text { Wavelet } \\
\text { Level-5 }\end{array}$ & 384 & 896 & 116 \\
\hline
\end{tabular}

\section{IMPLEMENTATION}

The method is implemented in Matlab 7.0 on Intel Core 2 Duo Processor T8100, $2.1 \mathrm{GHz}, 2$ GB RAM machine to obtain results. To check the performance of proposed technique we have used precision and recall. To test the proposed CBIR techniques using T-Wavelets, we have used database [11] of 1000 variable size images spread across 11 categories of human being, animals, natural scenery and manmade things. The sample images from the database are shown in figure 3 . Here all images were resized to $256 \times 256 \times 3$ before using T-Wavelets for feature extraction. Five queries were selected from each category of images, so in all 55 queries for every $\mathrm{T}$-Wavelet level and complete transform are fired on the database to test the proposed CBIR techniques.

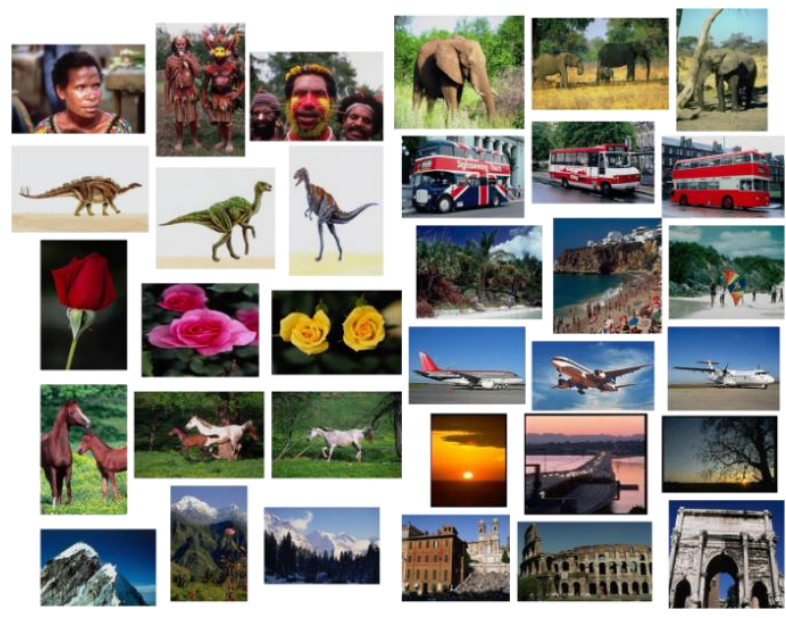

Figure. 3. Sample images from first database, the database has 11 categories, for a total of 1,000 images

In [12] Jain et al. address some of the features of an efficient CBIR system such as accuracy, stability and speed. To measure the retrieval effectiveness, we have used the precision and recall as statistical comparison parameters for our proposed technique of CBIR. The standard definitions of these two measures are given by following equations.

$$
\begin{aligned}
& \operatorname{Pr} \text { ecision }=\frac{\text { Number_of_relevant_images_retrieved }}{\text { Total_number_of_images_retrieved }} \\
& \operatorname{Re} \text { call }=\frac{\text { Number_of_relevant_images_retrieved }}{\text { Total_number_of_relevent_images_in_database }}
\end{aligned}
$$

\section{RESULTS AND DISCUSSIONS}

The query and database image matching is done using Euclidian distance for Gray-T-Wavelets and Color-T-Wavelets. The average precision and recall values are computed by grouping the number of retrieved images.

The crossover point of precision and recall for CBIR techniques acts as an important parameters [32] to judge its performance.

Figure 4 shows crossover of precision and recall for Gray-Walsh Wavelet and figure 5 shows it for RGB-Walsh Wavelet. Figure 4, 5 gives the crossover of precision and recall plotted against Number of retrieved images for Gray-Walsh Wavelet and RGBWalsh Wavelet compared with Complete Walsh transform Here the crossover point of Walsh Wavelet level-5 is the highest (0.332 in Gray-Walsh Wavelet and 0.401 in RGB-Walsh Wavelet) showing the best performance for CBIR as compared to complete Walsh transform and Walsh Wavelets of other levels. Overall the crossover point values in RGB-Walsh Wavelet are greater than the respective crossover point values in Gray-Walsh Wavelet. The precision/recall values and crossover points proves that the discrimination capability of Walsh Wavelet in CBIR is better than Walsh transform based CBIR and Walsh Wavelet of level 5 gives the best performance in all. 


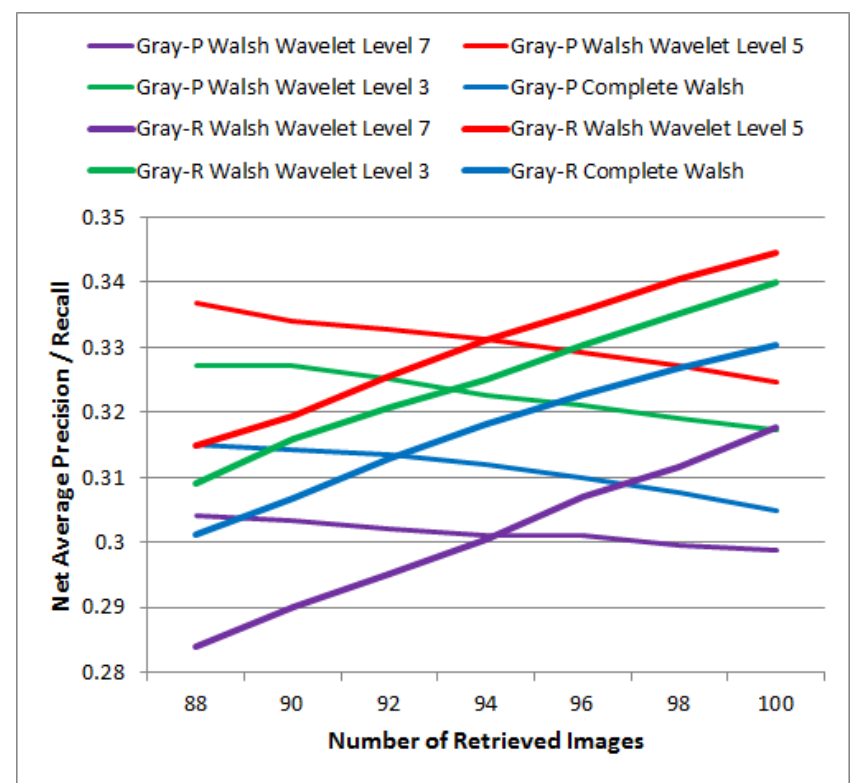

Figure 4 Crossover points of Precision-Recall for Gray-Walsh Wavelet

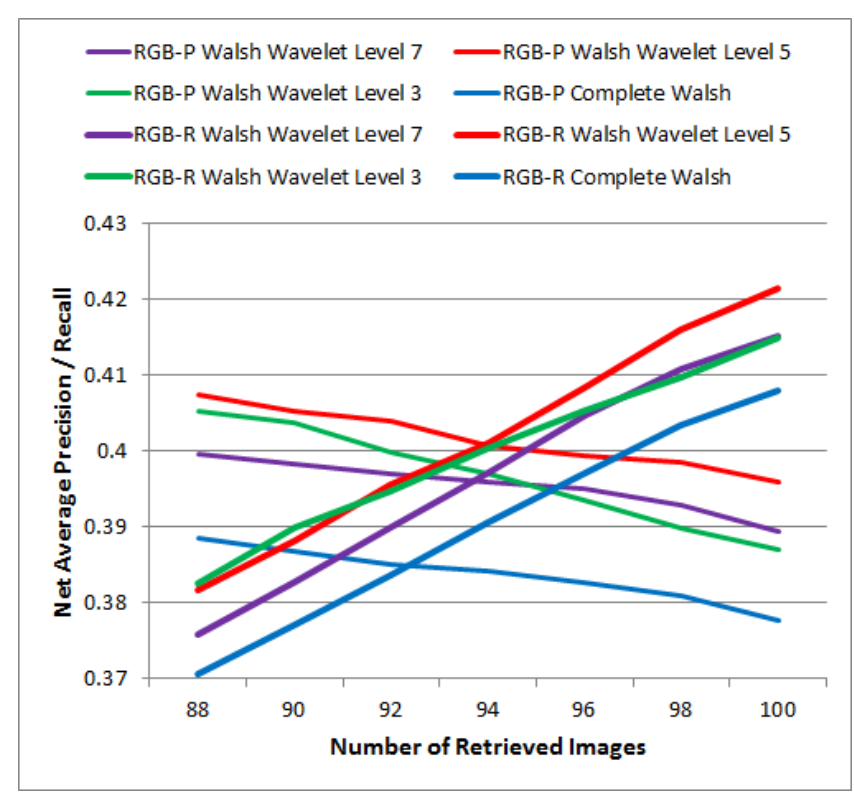

Figure 5 Crossover points of Precision-Recall for RGB-Walsh Wavelet

Figure 6 shows crossover of precision and recall for Gray-Haar Wavelets and figure 7 shows for RGB-Haar Wavelets. Here the crossover point of Haar Wavelet level-5 is the highest (0.32 in Gray-Haar Wavelet and 0.4 in RGB-Haar Wavelet) showing bettter performance as compared to complete Haar transform and Haar wavelets of other levels. Overall the crossover point values in RGB-Haar Wavelets are greater than the respective crossover point values in Gray-Haar Wavelets. The precision/recall values and crossover points proves that the discrimination capability of Haar Wavelets in CBIR is better than Haar transform based CBIR and Haar Wavelet of level 5 gives the best performance in all.

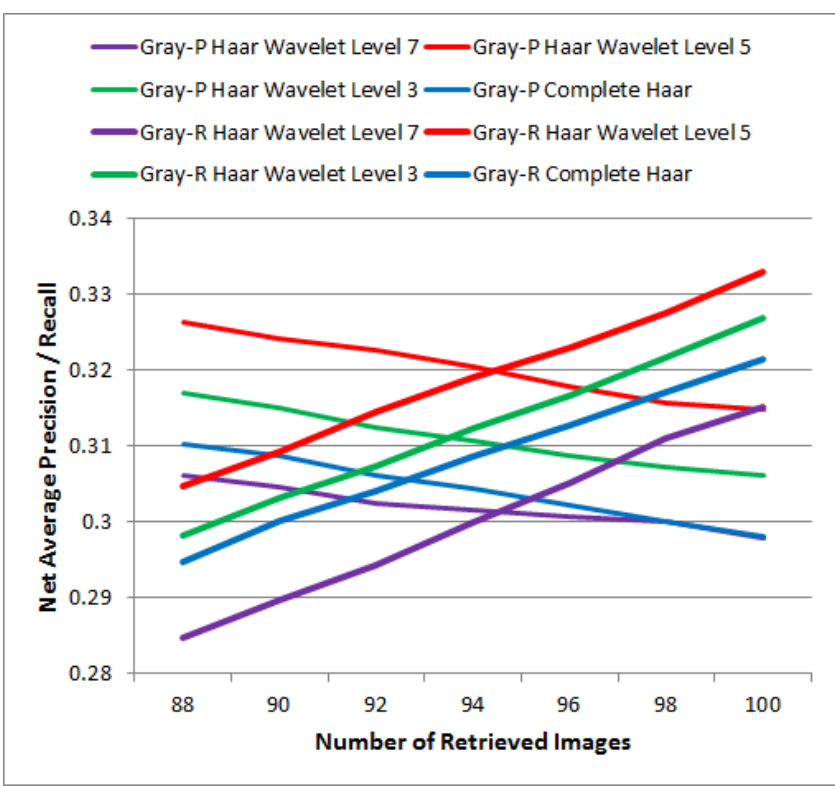

Figure 6 Crossover points of Precision-Recall for Gray-Haar Wavelet

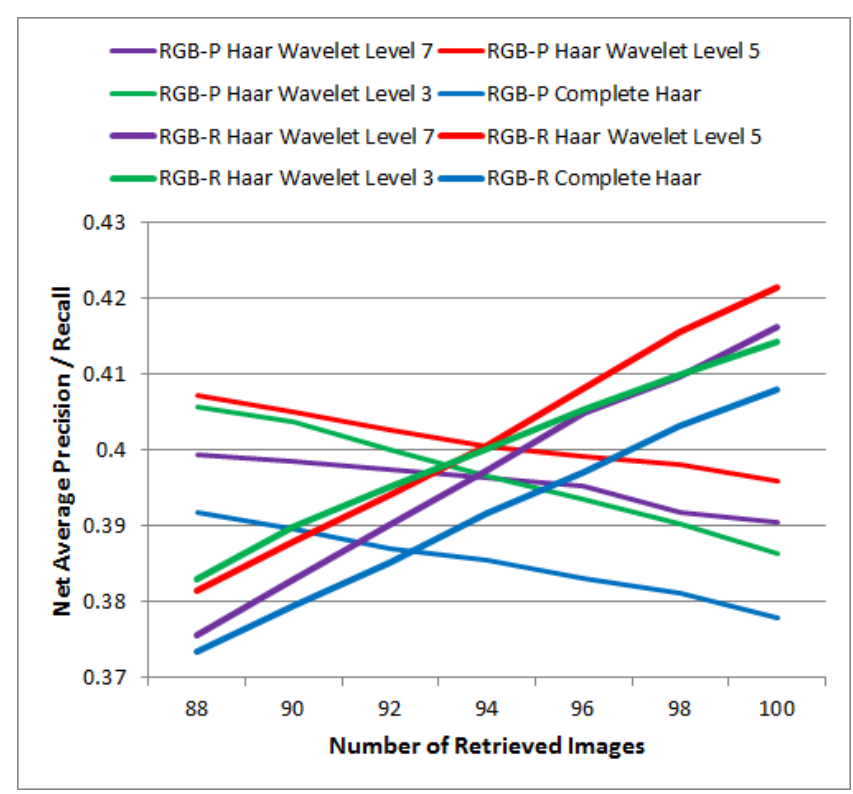

Figure 7 Crossover points of Precision-Recall for RGB-Haar Wavelet

Figure 8 shows crossover of precision and recall for Gray-Kekre Wavelets and figure 9 shows it for RGB-Kekre Wavelets. Here Kekre transform is giving better performance than Kekre Wavelets for gray and RGB plane. 


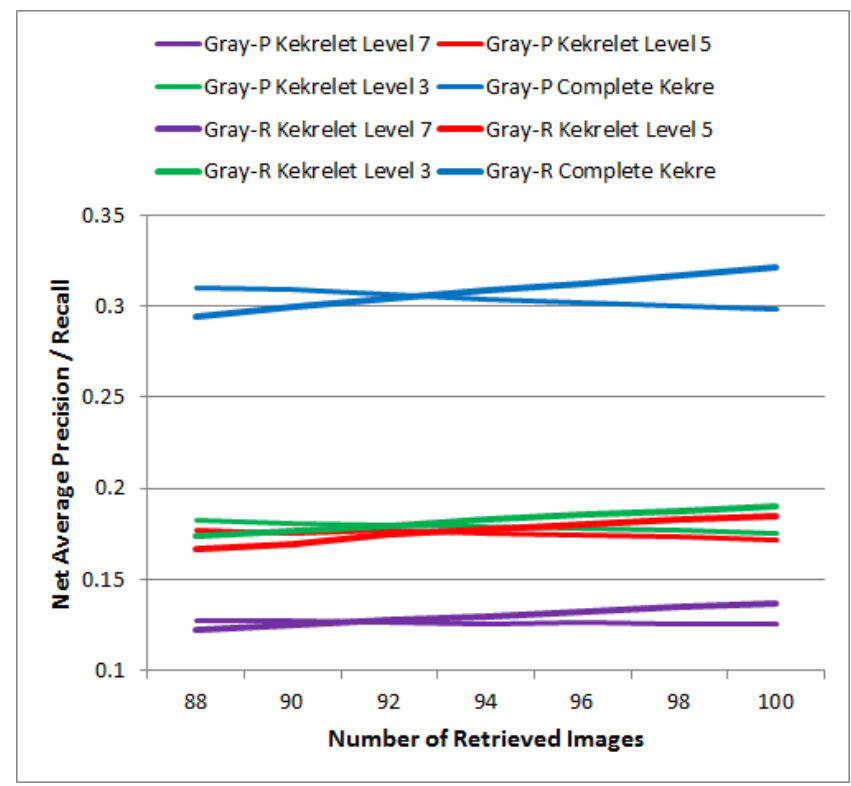

Figure 8 Crossover points of Precision-Recall for Gray-Kekre Wavelet

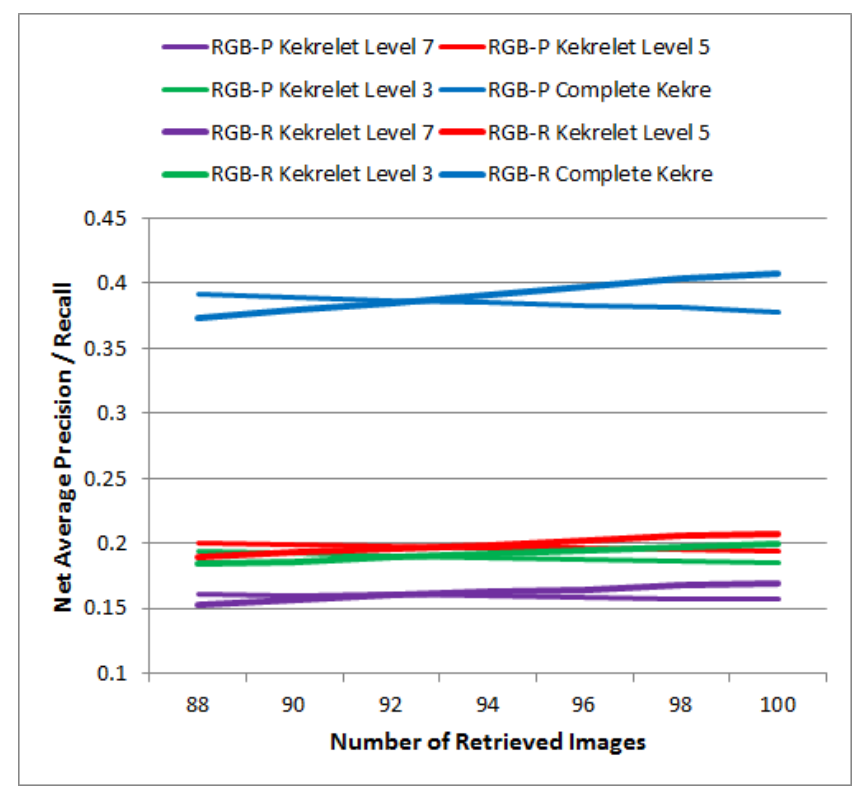

Figure 9 Crossover points of Precision-Recall for RGB-Kekre Wavelet

Figure 10 shows that out of all discussed Wavelets, Walsh Wavelets gives the best performance for gray images with the highest crossover point at level-5.

Figure 11 shows that out of all discussed Wavelets, Haar Wavelets give better performance till level-2 and then Walsh Wavelets take over till level-6, with the highest crossover points at level-6.

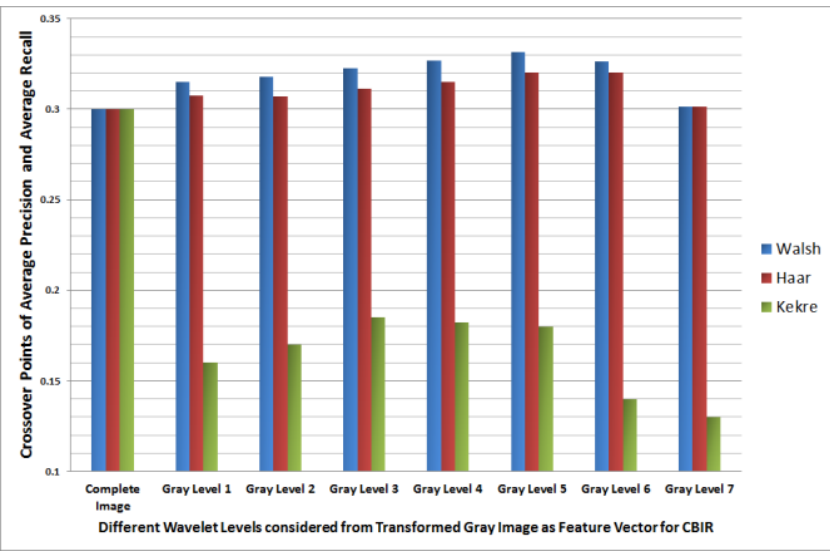

Figure 10 Crossover points of Precision-Recall for Walsh, Haar and Kekre Wavelets at different levels for gray images

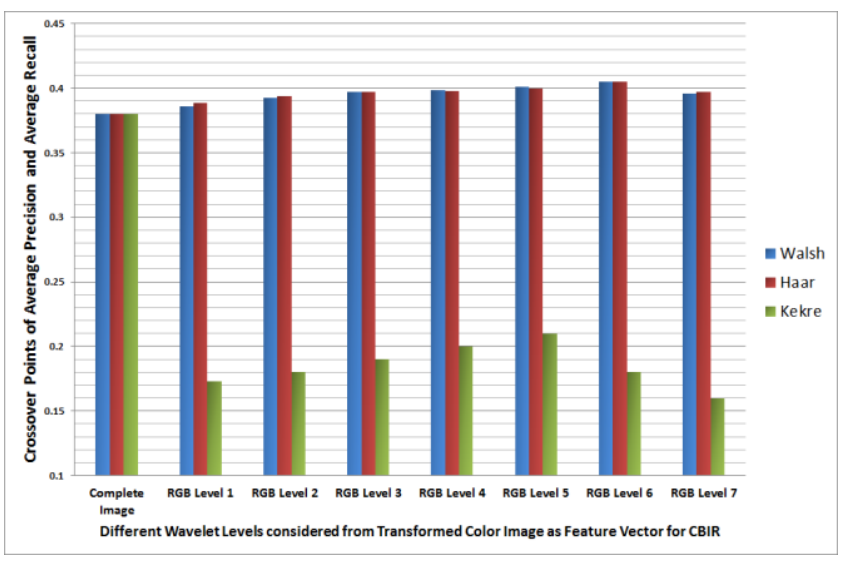

Figure 11 Crossover points of Precision-Recall for Walsh, Haar and Kekre Wavelets at different levels for color images

\section{CONCLUSION}

For finding similar images more precise and faster retrieval techniques are needed to access the large image archives generated. In this paper the Wavelet Pyramid based image retrieval techniques have been analyzed using Walsh, Haar and newly introduced Kekre's wavelet transforms. The Gray-Wavelet and Color-Wavelet based CBIR techniques are tested using 55 queries fired on the image database with 1000 images spread over 11 categories. The precision/recall values show that Walsh and Haar Wavelet Pyramid based image retrieval techniques outperform complete Walsh and Haar transform based image retrieval proving that Wavelet Pyramid gives better discrimination capability in Walsh and Haar Wavelets but fails to do the same in Kekre Wavelet transform as indicated by lower precision and recall values. But the advantage of Kekre wavelet transform over others is tremendous speed of feature extraction as indicated in table 1. In all Wavelet of level-5 gives better performance than other Wavelets in the considered transforms. The goal of improving performance of image retrieval technique with reduced complexity is achieved by Haar and Walsh Wavelet Pyramid.

\section{REFERENCES}

[1] H.B.Kekre, Sudeep D. Thepade, "Image Retrieval using Color-Texture Features Extracted from Walshlet Pyramid", 
ICGST International Journal on Graphics, Vision and Image Processing (GVIP), Volume 10, Issue I, Feb.2010, pp.9-18, Available online www.icgst.com/gvip/Volume10/Issue1/P1150938876.html.

[2] H.B.Kekre, Sudeep D. Thepade, "Image Retrieval using Augmented Block Truncation Coding Techniques", Proc. ACM Int. Conf. on Advances in Computing, Communication and Control (ICAC3-2009), 23-24 Jan 2009, Fr. Conceicao Rodrigous College of Engg., Mumbai. Is uploaded and available online at ACM portal.

[3] H.B.Kekre, Sudeep D. Thepade, "Rendering Futuristic Image Retrieval System", Proc. National Conf. on Enhancements in Computer, Comm. and Info. Technology, EC2IT2009,20-21 Mar 2009, K.J. Somaiya COE, Vidyavihar, Mumbai-77.

[4] H.B.Kekre, Sudeep D. Thepade, "Color Traits Transfer to Grayscale Images", IEEE -International Conference on Emerging Trends in Engineering and Technology, ICETET2008, 16-18 July 2008, Raisoni College of Engineering, Nagpur. Is uploaded and available online at IEEE Xplore CSDL, ACM Portal

[5] H.B.Kekre, Sudeep D. Thepade, "Image Blending In Vista Creation using Kekre's LUV Color Space" , SPIT -IEEE Colloquium and International Conference, 04-05 Feb 2008, SPIT Andheri ,Mumbai.

[6] K.-C. Liang and C. C. Kuo, "WaveGuide: A Joint WaveletBased Image Representation and Description System," IEEE Trans. on Image Processing, vol. 8, no. 11, pp.1619-1629, 1999

[7] W. Y. Ma, B. S. Manjunath, "A comparison of wavelet features for texture annotation," Proc. of IEEE Int. Conf. on Image Processing, Vol. II, pp. 256-259, Washington D.C., Oct. 1995.

[8] Hirata K. and Kato T. "Query by visual example - contentbased image retrieval", In Proc. of Third International Conference on Extending Database Technology, EDBT'92, 1992, pp 56-71.

[9] Haar, Alfred, "Zur Theorie der orthogonalen Funktionen systeme". (German), Mathematische Annalen, volume 69, No. 3, 1910, pp. 331-371.

[10] Charles K. Chui, “An Introduction to Wavelets”, Academic Press, 1992, San Diego, ISBN 0585470901.

[11] http://wang.ist.psu.edu/docs/related/Image.orig (last referred on June, $10^{\text {th }}, 2009$ )

[12] A. K. Jain , A. Vailaya, "Image Retrieval using Colour and Shape," In Proc. of 2nd Asian Conference on Computer Vision (ACCV-95), Singapore, 1995, pp. 529-533.

[13] H.B.Kekre, Sudeep D. Thepade, "Ubicomp The Future of Computing Technology", Techno Path : Journal of Science Technology and Management, Volume 1, Issue 2, 2009.

[14] Robert Li, Jung Kim, "Image Compression Using Fast Transformed Vector Quantization", IEEE Applied Imagery Pattern Recognition Workshop, 2000 Proceedings, Volume 29, 2000, pp.141- 145.

[15] B.G.Prasad, K.K. Biswas, and S. K. Gupta, "Region -based image retrieval using integrated color, shape, and location index”, International Journal on Computer Vision and Image Understanding Special Issue: Colour for Image Indexing and Retrieval, Vol. 94, Issues 1-3, April-June 2004, pp.193-233.

[16] Minh N. Do, , and Martin Vetterli, , "Wavelet-Based Texture Retrieval Using Generalized Gaussian Density and KullbackLeibler Distance", IEEE Transactions On Image Processing, Volume 11, Number 2, pp.146-158, February 2002.

[17] A. Gupta, R. Bach, C. Fuller, A. Hampapur, B. Horowitz, R. Jain, C.F. Shu "The Virage image search engine: an open framework for image management" in Storage and Retrieval for Image and Video Databases IV, Proc SPIE Vol. 2670, pp 76-87, 1996.

[18] M. Flickner, H. Sawhney, W. Niblack, J. Ashley, Q. Huang, B. Dom, M. Gorkani, J. Hafner, D. Lee, D. Petkovic, D. Steele, and P. Yanker. "Query by image and video content: The QBIC system," IEEE Computer, vol. 28, pp. 23-32, 1995.

[19] H.B.Kekre, Sudeep D. Thepade, "Boosting Block Truncation Coding using Kekre's LUV Color Space for Image Retrieval", WASET International Journal of Electrical, Computer and System Engineering (IJECSE), Volume 2, Number 3, Summer 2008. Available online at http://www.waset.org/ijecse/v2/v2-3-23.pdf

[20] M. La Cascia, S. Sethi, S. Sclaroff. "Combining textual and visual cues for content-based image retrieval on the world wide web", In IEEE Workshop on Content-based Access of Image and Video Libraries, pp 24-28, Santa Barbara, CA, June 1998.

[21] Carson M. Thomas, S. Belongie, J. M. Hellerstein, and J. Malik, "Blobworld: a system for region-based image indexing and retrieval", In Visual Information and Information Systems (VISUAL), LNCS 1614, pages 509516, Amsterdam, The Netherlands, June 1999.

[22] S. Sclaroff, L. Taycher, and M. La Cascia, "Image Rover: a content-based image browser for the world wide web", In IEEE Workshop on Content-based Access of Image and Video Libraries, pp. 2-9, San Juan, Puerto Rico, June 1997.

[23] H.B.Kekre, Tanuja K. Sarode, Sudeep D. Thepade, "Image Retrieval using Color-Texture Features from DCT on VQ Codevectors obtained by Kekre's Fast Codebook Generation", ICGST International Journal on Graphics, Vision and Image Processing (GVIP), Volume 9, Issue V, Sept. 2009, pp. 1-8, Available onlineathttp://www.icgst.com/gvip/Volume9/Issue5

[24] Zaher Al Aghbari and Ruba Al-Haj, "Building SSeg-Tree for Image Representation and Retrieval", ICGST Int. Journal on Graphics, Vision and Image Processing (GVIP), Special Issue on Image Retrieval and Representation, Vol. 6, Year 2006, pp. 101-109.

[25] M.Eisa, I.Elhenawy, A.E.Elalafi and H. Burkhardt, "Image Retrieval based on Invariant Features and Histogram Refinement", ICGST Int. Journal on Graphics, Vision and Image Processing (GVIP), Special Issue on Image Retrieval and Representation, Vol. 6, Year 2006, pp. 7-11.

[26] H.B.Kekre, Sudeep D. Thepade, "Color Based Image Retrieval using Amendment Block Truncation Coding with YCbCr Color Space", International Journal on Imaging (IJI), 
Volume 2, Number A09, Autumn 2009, pp.2-14. Available online at www.ceser.res.in/iji.html

[27] H.B.kekre, Tanuja K. Sarode, Sudeep D. Thepade, "ColorTexture Feature based Image Retrieval using DCT applied on Kekre's Median Codebook", International Journal on Imaging (IJI), Volume 2, Number A09, Autumn 2009,pp. 5565. Available online at www.ceser.res.in/iji.html

[28] Tuceryan M., Jain A.K.,"Texture Analysis Handbook of Pattern Recognition and Computer Vision (Eds. C.H.Chen, L.F.pau, P.S.P.Wang), 1994.

[29] H.B.Kekre, Sudeep D. Thepade, Archana Athawale, Anant Shah, Prathmesh Verlekar, Suraj Shirke, "Walsh Transform over Row Mean and Column Mean using Image Fragmentation and Energy Compaction for Image Retrieval", International Journal on Computer Science and Engineering (IJCSE), Volume 2S, Issue1, January 2010, (ISSN: 09753397). Available online at www.enggjournals.com/ ijcse.

[30] H.B.kekre, Sudeep D. Thepade, "Improving "Color to Gray and Back' using Kekre's LUV Color Space”, IEEE International Advanced Computing Conference 2009 (IACC'09), Thapar University, Patiala, INDIA, 6-7 March 2009. Is uploaded and available online at IEEE Xplore.

[31] H.B.Kekre, Sudeep D. Thepade Akshay Maloo, "Query by Image Content using Color-Texture Features Extracted from Haarlet Pyramid", International Journal Computer Applications (IJCA), Special issue on Computer Aided Soft Computing Techniques for Imaging and Biomedical Applications, August 2010.

[32] H.B.Kekre, Sudeep D. Thepade, Akshay Maloo, "Query by Image Content Using Colour Averaging Techniques", for International Journal of Engineering Science and Technology (IJEST), Volume 2, Issue 6, 2010. Available online at http://www.ijest.info.

\section{Author Biographies}

Dr. H. B. Kekre has received B.E. (Hons.) in Telecomm. Engineering. from Jabalpur University in 1958, M.Tech (Industrial Electronics) from IIT Bombay in 1960, M.S.Engg. (Electrical Engg.) from University of Ottawa in 1965 and Ph.D. (System Identification) from IIT Bombay in $1970 \mathrm{He}$ has worked as Faculty of Electrical Engg. and then HOD Computer Science and Engg. at IIT Bombay. For 13 years he was working as a professor and head in the Department of Computer Engg. At Thadomal Shahani Engineering. College, Mumbai. Now he is Senior Professor at MPSTME, SVKM's NMIMS University. He has guided $17 \mathrm{Ph} . \mathrm{Ds}$, more than 100 M.E./M.Tech and several B.E./B.Tech projects. His areas of interest are Digital Signal processing, Image Processing and Computer Networking. He has more than 320 papers in National / International Conferences and Journals to his credit. He was Senior Member of IEEE. Presently He is Fellow of IETE and Life Member of ISTE Recently nine students working under his guidance have received best paper awards. Currently 10 research scholars are pursuing Ph.D. program under his guidance.

Sudeep D. Thepade has Received B.E.(Computer) degree from North Maharashtra University with Distinction in 2003. M.E. in Computer Engineering from University of Mumbai in 2008 with Distinction, currently pursuing Ph.D. from SVKM's NMIMS, Mumbai. He has about than 07 years of experience in teaching and industry. He was Lecturer in Dept. of Information Technology at Thadomal Shahani Engineering College, Bandra(w), Mumbai for nearly 04 years. Currently working as Associate Professor in Computer Engineering at Mukesh Patel School of Technology Management and Engineering, SVKM's NMIMS University, Vile Parle(w), Mumbai, INDIA. He is member of International Association of Engineers (IAENG) and International Association of Computer Science and Information Technology (IACSIT), Singapore. His areas of interest are Image Processing and Computer Networks. He has about 75 papers in National/International Conferences/Journals to his credit with a Best Paper Award at International Conference SSPCCIN-2008, Second Best Paper Award at ThinkQuest-2009 National Level paper presentation competition for faculty and Best Paper Award at Springer International Conference ICCCT-2010.

Akshay Maloo is currently pursuing B.Tech. (CS) from MPSTME, NMIMS University, Mumbai. His areas of interest are Artificial intelligence, Image Processing, Computer Networks and Databases. $\mathrm{He}$ has 11 papers in National/International Conferences/Journals to his credit. 